Article

\title{
Bioactive Compounds from Monotheca Buxifolia Inhibit the Growth of Hepatocellular Carcinoma Cells
}

\author{
Said Hassan ${ }^{1,2,3,3,}$, Ashfaq Ur Rehman ${ }^{1, \#}$, , Syed Babar Jamal ${ }^{4}$, Yaqin Liu ${ }^{5, *}$ and Shaoyong Lu 1,* \\ 1 Department of Pathophysiology, Key Laboratory of Cell Differentiation and Apoptosis of Chinese Ministry \\ of Education, Shanghai Jiao Tong University School of Medicine, Shanghai 20025, China \\ 2 School of Pharmacy, Tongji Medical College, Huazhong University of Science and Technology, Wuhan \\ Hubei 430030, China \\ 3 Center of Biotechnology and Microbiology, University of Peshawar KPK, Pakistan \\ 4 Institure of Chemical Sciences, Gomal University D.I Khan KPK, Pakistan \\ 5 Medicinal Chemistry and Bioinformatics Center, Shanghai Jiao Tong University School of Medicine, \\ Shanghai 20025, China \\ \# These authors contribute equally to this manuscript. \\ * Correspondence: lushaoyong@yeah.ent (S.L.); liuyaqing7811@126.com (Y.L.)
}

\begin{abstract}
The natural products and conventional chemotherapeutic drugs are believed to increase the cure rates of anti-cancer treatment while reducing their toxicity. The current study investigates the cytotoxic and apoptogenic effects of bioactive compounds from Monotheca buxifolia on Hep G2 cell lines. The effect on the viability of Hep G2 cells was evaluated by MTT assay; Morphological changes were studied, the apoptotic activity was demonstrated through Annexin-V-FITC/ PI, a molecular dynamics simulation study was conducted to explore the binding pattern of the compounds in the active site of the PPRAS protein. The isolated compounds lauric acid, oleanolic acid, and bis(2-ethylhexyl) phthalate inhibited the growth of hepatocellular cancer cells, as determined by MTT assay and annexin V-FITC/PI. The IC 50 value for lauric acid was $56.4584 \pm 1.20$ $\mu \mathrm{g} / \mathrm{ml}$, that for oleanolic acid was $31.9421 \pm 1.03 \mu \mathrm{g} / \mathrm{ml}$, and that for bis(2-ethylhexyl) phthalate was $83.8019 \pm 2.18 \mu \mathrm{g} / \mathrm{ml}$. After $24 \mathrm{~h}$ of treatment, $29.5 \%$ of Hep G2 cells treated with lauric acid, $52.1 \%$ of those treated with oleanolic acid, and $22.4 \%$ of those treated with bis(2-ethylhexyl) phthalate were apoptotic. Morphological assay and Hoechst staining microscopy revealed the morphological alterations of cell membrane accompanied by nuclear condensation after treatment. The high fluctuation indicates the high potency and adopting various interactions, and vice versa, the oleanolic acid showed highly residues fluctuation, which remains stable in the active site of PPAR $\delta$ protein and involved in various interactions while remaining locally fluctuated in the binding site the other two compounds. In conclusion, a significant apoptogenic effect was exhibited by lauric acid, oleanolic acid, and bis(2-ethylhexyl) phthalate against HepG2 cells in inducing apoptosis. Our findings indicate that these bioactive compounds hold promise as potential therapeutic for hepatocellular carcinoma.
\end{abstract}

Keywords: HepG2 cells; cytotoxicity; apoptosis; Hoechst staining; molecular dynamics

\section{Introduction}

Natural resources such as plants, microbes, vertebrates, and invertebrates, are valuable sources of bioactive compounds. A large number of drugs have been developed in medicinal practice from natural products [1]. Many studies are currently being carried out to identify and characterize substances found in nature. Natural products are compounds or materials that could be acquired from living organisms, including animals, plant life, insect, venom, and microorganism [2]. 
Biologically active ingredients of therapeutics, commercials, and toxic properties have been explored all over and have many secondary metabolite ingredients [3]. It is estimated that about $50 \%$ of the drugs have been acquired from herbal products [4].

Most cancers involve uninhibited cell division that proliferates in free or normal cells of the body and eventually harms the surrounding tissues, leading to organs malfunctioning, and ultimately, deaths $[5,6]$. Globally after heart disease, cancer is the leading cause of death, with an estimated ten million cases of various types of malignancy reported each year [7]. The toxicities of chemotherapeutics retailers to the brine shrimp naupli have straight correlations with human nasopharyngeal carcinoma [8]. In the beginning, advanced for the insecticides' ability of the natural products, the lethal dose toxicity activity is now the easiest, in a short time, a robust and globally acceptable procedure for the evaluation of anti-cancer capacity [9].

Hepatocellular carcinoma (HCC) affects all sectors of the world population. It is the fifth universal cancer among other types of cancers and has the second-highest mortality rate among all cancers [10]. The incidence of deaths among HCC patients is rising gradually, with 746,000 deaths each year around the globe [11]. These account for approximately 78,200 new cases per year [12]. In 2017, 40,710 new cases of HCC were estimated, of which male cases being 29,200, in the United States of America (USA) alone. Furthermore, some deaths due to liver cancer estimated in 2017 were 28,920 $(19,610$ males; 9310 females) in the USA [13].

Monotheca buxifolia species founds in hilly areas of Pakistan which has therapeutic uses such as antiseptic, relieving gastrointestinal disorders, analgesic, anti-inflammatory, antipyretic, and wound healings [14]. These plants are found in abundance in Dir Lower, Attock, Xhob Kala Chitta, and Chitral [15]. In this study, cytotoxic, apoptotic activities, and computational studies of Lauric acid, Oleanolic acid, and Bis(2-ethylhexyl) phthalate bioactive compounds from Monotheca buxifolia were carried out, which might lead to potential management of hepatocellular carcinoma.

\section{Materials and Methods}

\subsection{Plant collection}

Aerial parts of Monotheca buxifolia (MB) was collected from, District Dir Lower, Khyber Pakhtunkhwa-Pakistan. Dr. Jehandar Shah, the Taxonomist, Ex-Vice Chancellor Shaheed Benazir University Shiringal, Pakistan, authenticated the plant's samples. The plants were cleaned with tap water thoroughly and rinsed with distilled water. Plants materials were dried under shade at room temperature. Next, the plant was chopped to make powder.

\subsection{Extraction and isolation}

Dried plants were chopped and cut down into pieces and grounded into fine powder form by using an electric grinder. The powder materials of Monotheca Buxifolia (15 kg each) were soaked three times in methanol for 15 days with random shaking at room temperature after completion of immersing the methanolic soluble portion was filtered. The concentration of the filtrates was carried out by using a rotary evaporator under $40^{\circ} \mathrm{C}$, and the blackish methanolic extract was obtained. 1000 g obtained from Monotheca buxifolia. $\mathrm{MeOH}$ extract $(900 \mathrm{~g})$ was suspended in water and successively partitioned with hexane, DCM, and EtOAc. The EtOAc fraction $(90 \mathrm{~g})$ was subjected to Column chromatography on silica gel (Merck Silica gel $60(0.063-0.200 \mathrm{~mm}), 5 \times 60 \mathrm{~cm})$. The column was first eluted with hexane-EtOAc (100:0 $\rightarrow 0: 100)$ as a solvent system. A total of 30 fractions, SH-1 to SH-30, were obtained based on TLC profiles. Lauric acid, oleanolic acid, and bis(2-ethylhexyl) phthalate were isolated from the subsequent fractions and further characterized by NMR (Figure 1). 

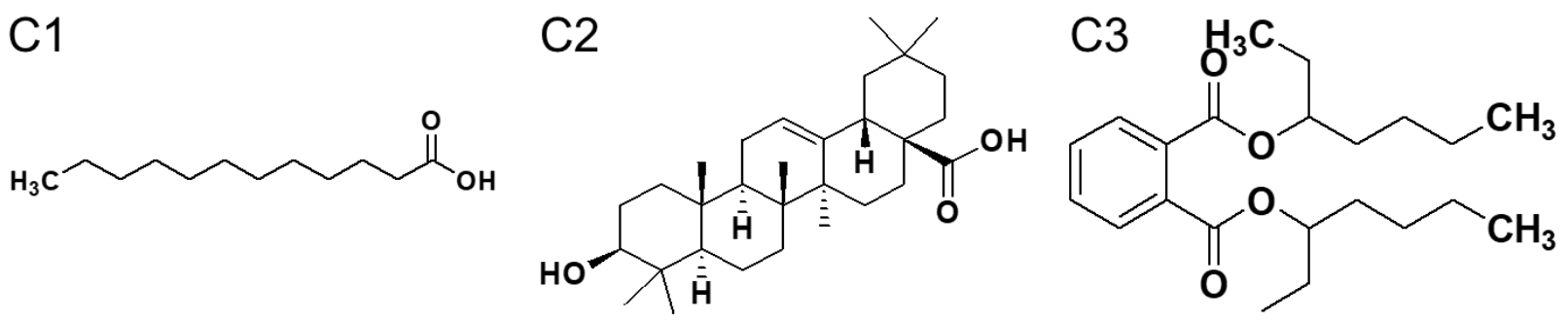

Figure 1. Chemicals Structures of C1 (Lauric Acid) C2 (Oleanolic acid) and C3 (Bis(2-ethylhexyl) phthalate) isolated from Monotheca buxifolia.

\subsection{Cytotoxic assay against Hep-G2 cells}

Hep G2 cells were collected by ATCC, Manassas, VA, USA. MTT procedure was used to assess cell death [16]. Briefly, 96-well plates were used to seed the cells of $5 \times 10^{4}$ cells $/ \mathrm{mL}$. Cells were seeded for $24 \mathrm{~h}$, After $24 \mathrm{~h}$, cells were treated with concentrations of $100 \mu \mathrm{g} / \mathrm{mL}$ and $75 \mu \mathrm{g} / \mathrm{mL}$ of Lauric acid, oleanolic acid, and Bis(2-ethylhexyl) phthalate and incubated for 48 hours. DMSO was treated as a negative control. After removal of the medium $1 \mathrm{mg} / \mathrm{mL}$, MTT reagents were added into each well. The incubation of plates was carried out for two hours at $37^{\circ} \mathrm{C}$ in $5 \% \mathrm{CO}_{2}$ atmosphere. MTT reagent was removed after incubation, and formazin violet products were dissolved in $100 \mu \mathrm{L}$ of DMSO. Sample absorbance was calculated by using microplate readers at a wavelength of $490 \mathrm{~nm}$. The readings obtained from samples were compared with control, whose viability is $100 \%$. The IC 50 $(\mu \mathrm{g} / \mathrm{mL})$ values of the cancerous cells were calculated. The experiments were performed in triplicates.

\subsection{Cell apoptosis study}

\subsubsection{Cell morphology}

Morphological observation of cells treated with lauric acid, oleanolic acid, and bis(2-ethylhexyl) phthalate was done to determine the changes induced by lauric acid, oleanolic acid, and bis(2ethylhexyl) phthalate. All the cells were exposed to increasing concentrations $(100 \mu \mathrm{g} / \mathrm{ml})$ of lauric acid, oleanolic acid, and bis(2-ethylhexyl) phthalate for $24 \mathrm{~h}$ and cell images were taken using an inverted phase-contrast microscope (OLYMPUS CKX 41) at $10 \mathrm{X}$ magnification [17].

\subsubsection{Hoechst 33258 staining assay}

Hoechst 33258 staining assay was carried out to evaluate cell apoptosis as previously reported [18] with minor modifications. HepG2 cells were seeded into 6-well plates overnight and then treated with $100 \mu \mathrm{g} / \mathrm{ml}$ of Lauric acid, oleanolic acid, and Bis(2-ethylhexyl) phthalate for $24 \mathrm{~h}$. The wells were then washed three times with PBS and fixed with $4 \%$ paraformaldehyde $(500 \mu \mathrm{L})$ for $30 \mathrm{~min}$. The cells were further washed three times with PBS, followed by staining with Hoechst $33258(500 \mu \mathrm{L}, 5$ $\mu \mathrm{g} / \mathrm{mL}$ ) for $10 \mathrm{~min}$ at $37^{\circ} \mathrm{C}$ in the dark. After being washed three times with PBS, the cells were visualized by a fluorescence microscope (IX71; Olympus, Japan).

\subsubsection{Annexin V-FITC/ PI assay}

Compounds isolated from Monotheca buxifolia were assessed for apoptotic study against Hep G2 cells. The apoptosis of the isolated compounds was calculated by flow cytometry. The amount of $2 \mathrm{x}$ 105 cells was cultured in $75 \mathrm{~cm} 2$ flasks with appended culture medium for 24 hours. Hep G2 cells were treated with $100 \mu \mathrm{g} / \mathrm{ml}$ of Lauric acid, oleanolic acid, and Bis(2-ethylhexyl) phthalate for $24 \mathrm{~h}$. The hydroalcoholic solution was used as a negative control. Anexin V/ PI assay was performed according to the protocol provided with the PI/FITC apoptosis kit. Hep-G2 cells were harvested, washed with PBS buffer solution, and kept in a sterilized tube. The cell pellets were suspended in $100 \mu \mathrm{L}$ of binding buffer. The cell suspensions were shifted to a micro-tube, to which $5 \mu \mathrm{L}$ of AnexinV/FITC conjugates and $10 \mu \mathrm{L}$ of propidium-iodide (PI) was added. The incubation of cells was carried out for 15 minutes in the dark at room temperature. Flow cytometry was used to determine the 
fluorescence of the cells [19]. Ten thousand cells were quantified by a flow cytometer (BD). BD Bioscience-C6 software was used to analyze the data. The experiment was performed in triplicate.

\subsection{Molecular dynamics simulation study}

To illustrate the binding mode of these selected compounds in the active pocket of peroxisome proliferator-activated receptors (PPAR), Molecular docking (MD) study was conducted using MOEDock ${ }^{\circledR}$ package [20]. The crystal structure of PPRAঠ (PDB ID 1i7i) [21] was retrieved from the protein data bank. Before MD, the 3D protonation and energy minimization (EM) of crystal structure was analyzed by using the default parameters of the MOE energy minimization algorithm (gradient: 0.05, Force Field: Amber99). All the selected compounds in the current study were downloaded from PubChem using PubChem ID 3893 for Lauric acid, 10494 for Oleanic acid, and 8343 for bis-(2ethylhexyl) phthalate, indicated by name lauric acid, oleanolic acid, and bis(2-ethylhexyl) phthalate thoroughly in the bioinformatics section. The EM for all the compounds was carried out up to 0.05 Gradient using the MMFF94s force field implemented in $\mathrm{MOE}^{\circledR}$. Finally, all the selected compounds were docked into the active site of protein utilizing the Triangular Matching docking method (default), and 100 different conformations for each compound have been generated. The predicted ligand-protein complexes were ranked by the scores from the GBVI/WSA binding free energy (BFE) calculation. The GBVI/WSA is a scoring function that estimates the BFE of the ligand from a given pose. For all scoring purposes, lower scores indicate a more favorable mode of interaction. The unit for all scoring purposes is $\mathrm{Kcal} / \mathrm{mol}$. The next top conformation of each compound was selected based on the docking score for further analysis. The predicted ligand-protein complexes were analyzed for molecular interactions in PyMol. More ever, the top conformer was further validated by molecular dynamics (MD) simulation study to explore the dynamic behaviors of the selected compounds in the active cavity. Total four systems were prepared for the MD simulation study; PPARס-APO, PPAR $\delta-$ C1, PPAR $-\mathrm{C} 2$, and PPAR $\delta-C 3$. The AMBER v14 tool was used to performed MD simulation; the topology of each compound was generated using the Antechamber module of AMBER. A 1.5-nm cubic box was solvated using the transferable intermolecular potential with 3 points (TIP3P) water model [22]. Sodium and chloride ions were added into the cubic box by the LEaP program to neutralize the overall systems. These ions had a maximum electrostatic potential to replace the water molecules. The energy was minimized for 6000 cycles using the steepest descent and conjugate gradient minimization for 3000 cycles [23]. Equilibration was performed with moles $(\mathrm{N})$, volume $(\mathrm{V})$, and energy (E) (NVT) ensemble at $300 \mathrm{~K}$, followed by NPT (amount of substance [N], pressure [P], and temperature [T]) for $300 \mathrm{~K}$ at a pressure of 1 bar for 2000 picosecond for each. The Berendsen thermostat method was applied for temperature [24] while pressure was maintained constant, and the bond length was rectified with the linear constraint solver algorithm.

\subsection{Statistical analysis}

The statistical analysis was done by Student's t-test using GraphPad Prism. The results were presented as Mean \pm SEM (standard error of the mean) of triplicates done in the same experiment or an average of three independent experiments $(n=3)$.

\section{Results and Discussion}

\subsection{Cytotoxic assay of the isolated compounds against Hep-G2 cells}

The effect of lauric acid, oleanolic acid, and bis(2-ethylhexyl) phthalate on the viability of laryngeal carcinoma cells was assessed by MTT assay. The (\%)-inhibition results from cell mortality after 48 hours' extracts incubation (Figure 2), a concentration of $100 \mu \mathrm{g} / \mathrm{ml}$ lauric acid shows $58.32 \pm$ $1.09 \%$, oleanolic acid shows $60.87 \pm 1.05 \%$ and Bis(2-ethylhexyl) phthalate shows $53.46 \pm 1.03 \%$ cytotoxic potency against Hep-2 cells while at concentration of $75 \mu \mathrm{g} / \mathrm{ml}$ lauric acid shows $54.27 \pm$ $0.98 \%$, oleanolic acid shows $57.65 \pm 1.06 \%$ and bis(2-ethylhexyl) phthalate shows $47.81 \pm 1.08 \%$ inhibition against Hep-2 cells. The IC 50 values of lauric acid is $56.4584 \pm 1.20 \mu \mathrm{g} / \mathrm{ml}$, oleanolic acid is $31.9421 \pm 1.03 \mu \mathrm{g} / \mathrm{ml}$ and bis(2-ethylhexyl) phthalate is $83.8019 \pm 2.18 \mu \mathrm{g} / \mathrm{ml}$ (Table 1). The following 
results further demonstrate that the cell lines were more sensitive to oleanolic acid as well as to other compounds. From the above study, all compounds have cytotoxic effects. Thus, these compounds could be used as a source for new lead structures in drug design to combat cancer [25].

Table 1. The IC 50 values of lauric acid, oleanolic acid, and bis(2-ethylhexyl) phthalate against Hep G2 cells.

\begin{tabular}{cc}
\hline Compounds & IC50 $\mathbf{\mu g} / \mathbf{m l}$ \\
\hline Lauric Acid (C1) & $56.4584 \pm 1.20$ \\
\hline Oleanolic acid (C2) & $31.9421 \pm 1.03$ \\
\hline Bis(2-ethylhexyl) phthalate (C3) & $83.8019 \pm 2.18$ \\
\hline
\end{tabular}

The active constituents, namely flavonoids and terpenoids, may be responsible for reducing the cancer risk factors [26]. The anti-cancer effect against liver cancer on Hep G2 cell lines showed that all three compounds possess an anti-proliferative effect.

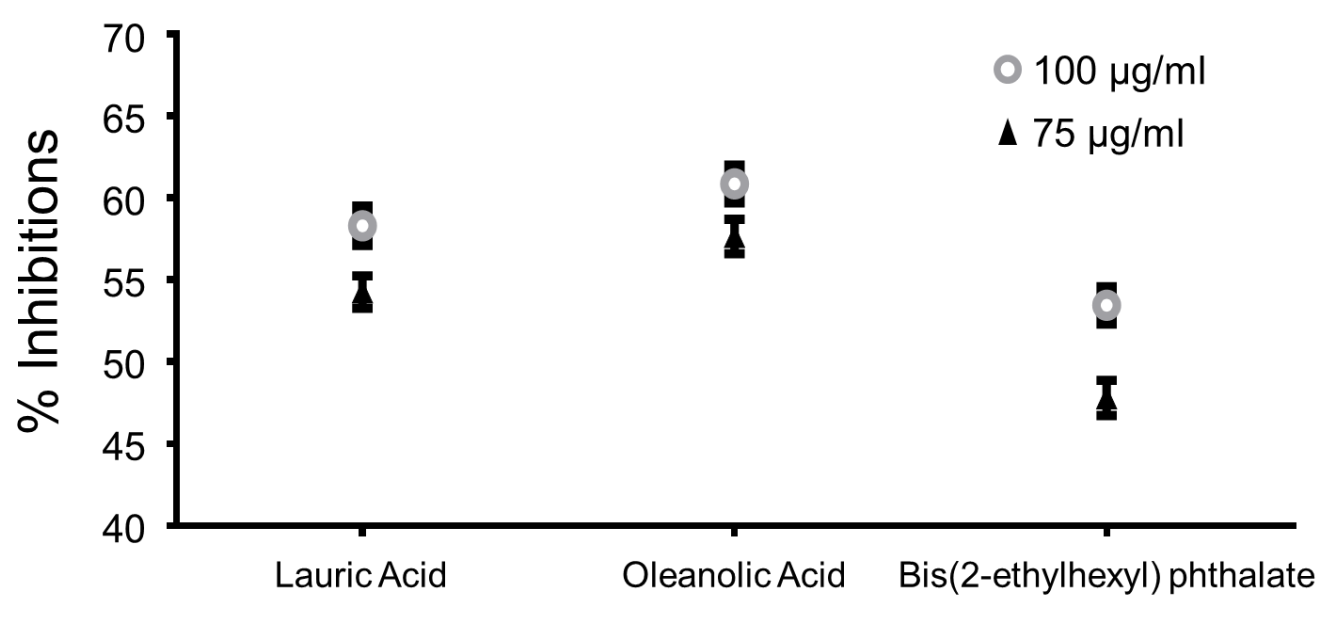

\section{Concentrations}

Figure 2. Hep G2 cytotoxicity of lauric acid, oleanolic acid, and bis(2-ethylhexyl) phthalate (Data are reported as mean \pm S.E.M).

\subsection{Cell apoptosis study}

\subsubsection{Morphological changes}

The morphological changes observed in Hep G2 cells are shown in Fig 3. Morphological alteration of Hep G2 Cells after exposure of lauric acid, oleanolic acid, and bis(2-ethylhexyl) phthalate was observed under a phase-contrast microscope. The cells indicated the most prominent effects after exposure to lauric acid, oleanolic acid, and bis(2-ethylhexyl) phthalate. Changes in morphology were observed in treated cells. Cells were exposed to $100 \mu \mathrm{g} / \mathrm{ml}$ lauric acid, oleanolic acid, and bis(2ethylhexyl) phthalate for $24 \mathrm{~h}$ reduced the morphology of the cells and cell adhesion capacity in comparison to control (Fig 3-5). Most of the cells lost their typical morphology and appeared smaller, shrunken and rounded. 

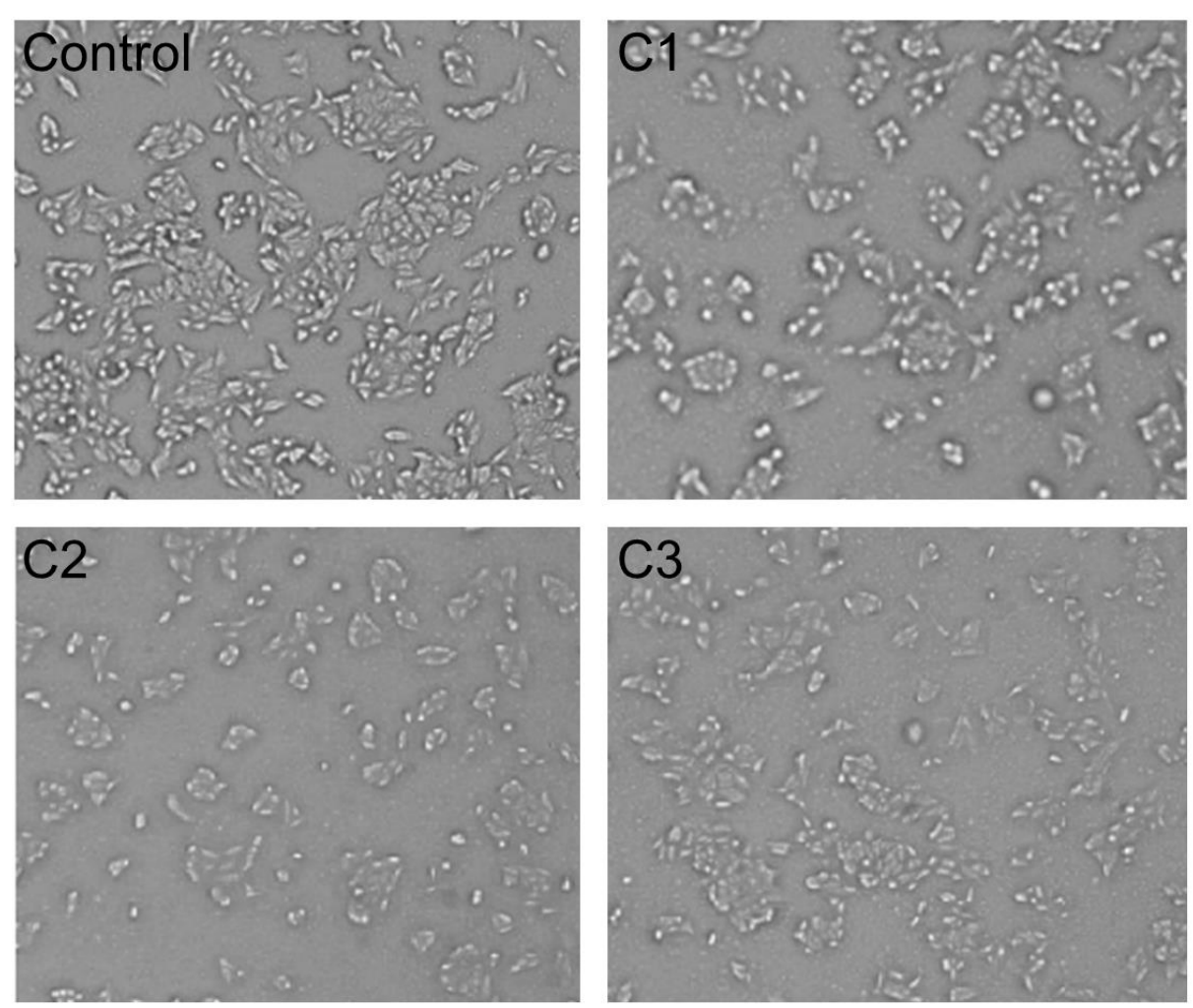

Figure 3. Morphological Changes in HepG2 Cells Following the exposure to C1 (Lauric acid), C2 (oleanolic acid), and C3 (bis(2-ethylhexyl) phthalate) after $24 \mathrm{~h}$ treatment. Images were taken using an inverted phase-contrast microscope at $10 \mathrm{X}$ magnification.

\subsubsection{Hoechst 33258 staining assay}

Staining cells with Hoechst 33258 is an ideal assay for distinguishing apoptotic cells from healthy or necrotic cells [27] because cells that have died by apoptosis will generally display condensed DNA and fragmented nuclei [28, 29], whereas healthy and necrotic cells do not. However, healthy cells undergoing mitosis may also have condensed DNA, and some cells can still die by apoptosis in the absence of nuclear fragmentation [30]. As shown in Fig 4, cells of the control group had normal nuclear morphology under a fluorescent microscope after Hoechst 33258 staining, indicating that the chromatin was equivalently distributed in the nucleus. The test group marked with nuclear fragmentation, condensation of chromatin, and the morphological characteristics of apoptosis, which include emitting brighter fluorescence, were detected after treatment with $100 \mu \mathrm{g} / \mathrm{ml}$ of Lauric acid, oleanolic acid and Bis(2-ethylhexyl) phthalate for $24 \mathrm{~h}$. These results indicated that Lauric acid, oleanolic acid, and Bis(2-ethylhexyl) phthalate are capable of inducing apoptosis in HepG2 cells. 

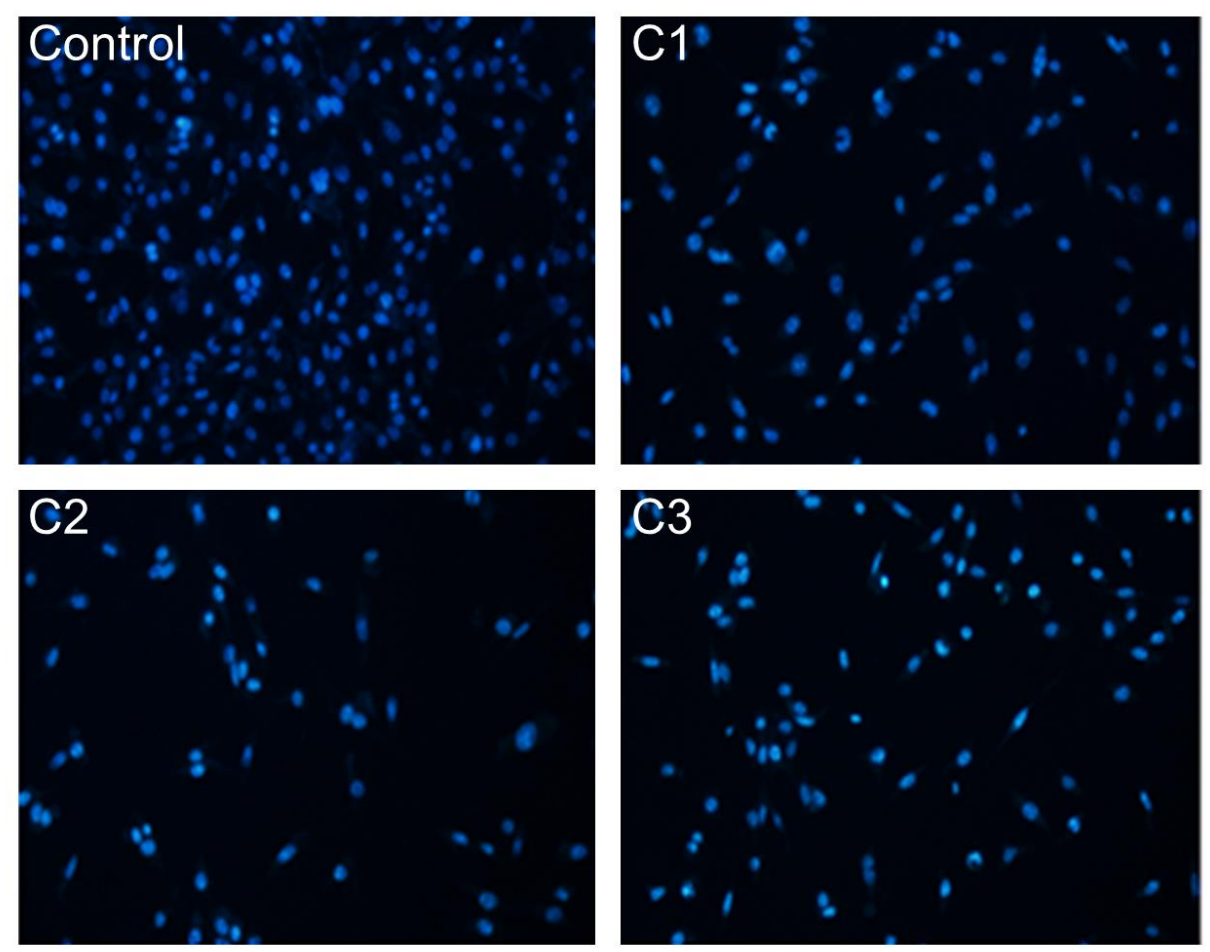

Figure 4. HepG2 cells treated with $100 \mu \mathrm{g} / \mathrm{ml}$ of C1 (Lauric acid), C2 (oleanolic acid), and C3 (bis(2ethylhexyl) phthalate) for $24 \mathrm{~h}$ were stained with nuclear stained (Hoechst 33258) and observed under fluorescent microscope 10×magnification.

\subsubsection{Annexin V-FITC/ PI assay}

Annexin V-FITC and PI staining assay is a way to detect the apoptotic level of cells. Cells were exposed to the concentration of $100 \mu \mathrm{g} / \mathrm{ml}$ of lauric acid, oleanolic acid, and bis(2-ethylhexyl) phthalate. After $24 \mathrm{~h}$, the percentage of apoptotic Hep G2 cells treated with lauric acid was $24.5 \%$, oleanolic acid was $51.4 \%$, and Bis(2-ethylhexyl) phthalate was $15.9 \%$ (Fig 5). A higher percentage of apoptotic cells were observed in wells treated with oleanolic acid than those treated with lauric acid and bis(2-ethylhexyl) phthalate. This result indicates that oleanoic acid has highly cytotoxic properties. Previously, oleanolic acid was testified to induce apoptosis in various cell lines [31-33]. The pharmaceutical industries are to derive a bioactive compound responsible for the cytotoxic effect with bioactive substances on cancer, on the other hand, cytoprotection of normal cells [33]. 

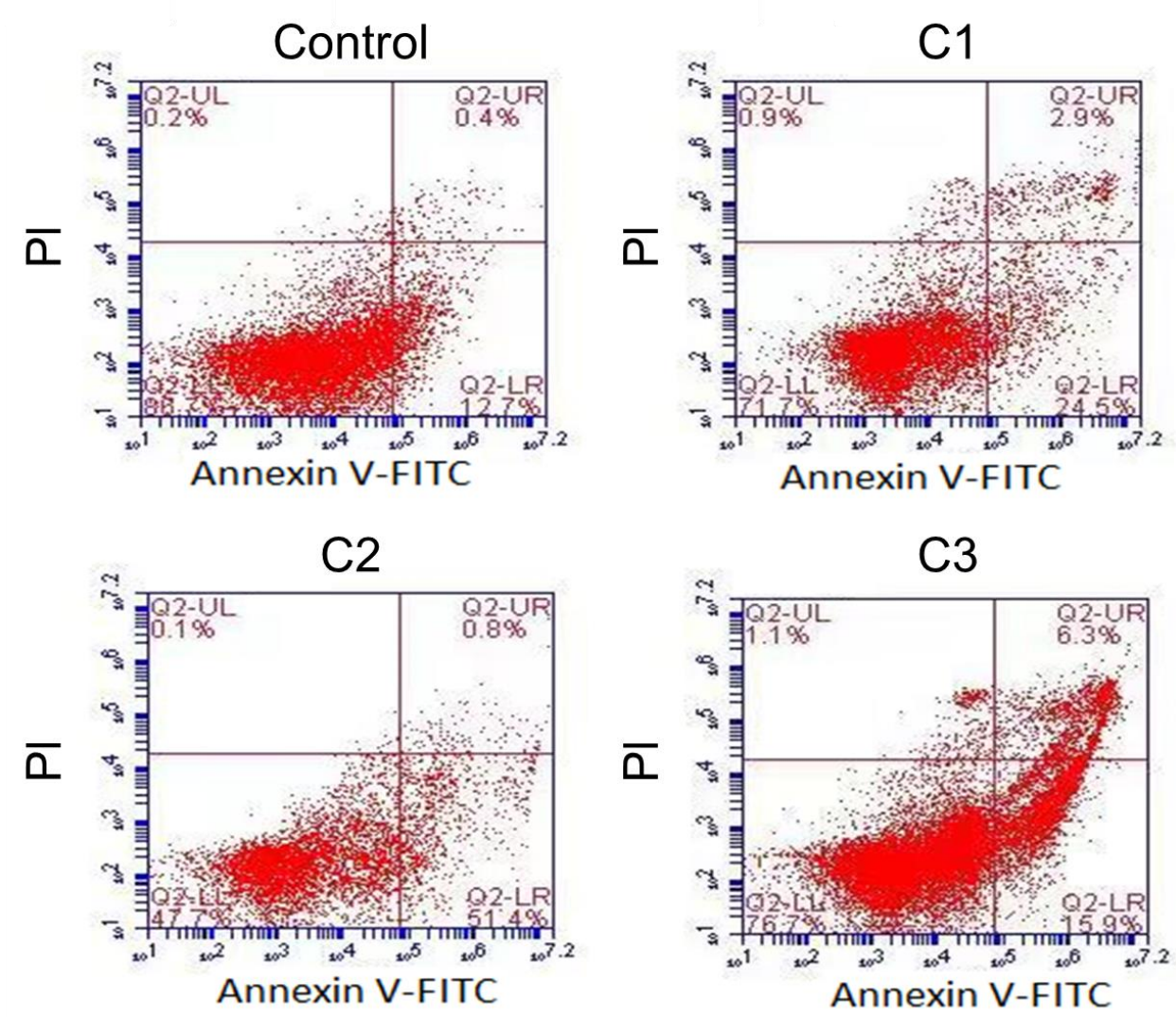

Figure 5. Annexin V and PI staining. C1 (Lauric acid), C2 (oleanolic acid) and C3 (bis(2-ethylhexyl) phthalate).

\subsection{Inspection of Stability of the Bound and Unbound Ligand Complex with PPRAS}

The molecular docking study revealed a fit-well pattern of binding by all the compounds in the active site, but solely differences observed in their mode of adopting interactions with active site residues of the PPRAठ protein. Furthermore, the molecular dynamics (MD) simulation study was conducted to illustrate the stability of all the compounds I complex with PPRAS protein. Subsequently, it was observed that the potent compound (PPRAס-C2) showed an energetic stabilized behavior in the binding site by adopting intermolecular interactions with active site residues, such as hydrogen bonds and hydrophobic interactions etc. In the present study, the binding mode for lauric acid, oleanolic acid, and bis(2-ethylhexyl) phthalate was investigated using molecular docking studies, and further validate these docked complexes through MD simulation study as shown in Fig 6. The results indicate that the compounds (lauric acid, oleanolic acid, and bis(2-ethylhexyl) phthalate) exhibit significant binding affinities in terms of adopting favorable interactions with active site residues of human PPARठ protein.

The MD simulation is one of the useful techniques to inspect the stability of docked proteinligand complexes concerning MD simulation time. MD simulations for each PPRAठ bound and unbound ligand complex was conducted via AMBER v14, to clearly illustrate the impact of the corresponding compound on overall PPRA protein structure. The root means square deviation (RMSd) was calculated based on the initial backbone coordinates of the protein-ligand complexes in order to evaluate the possible deviation in the structure during simulation, the RMS $d$ of all the PPRA $\delta$ bound and unbound ligand complexes relative to the original structures shows that the simulation time of total $50 \mathrm{~ns}$ is appropriate to reach equilibration at temperature $310 \mathrm{~K}$. We observed that the RMS $d$ of all PPRAঠ complexes showed a similar deviation till $5 \mathrm{~ns}$ but dramatically changed behavior observed, start from $5 \mathrm{~ns}$ onward. This point deviation pins the point of the potential impact of the various compound on the protein structure. The gradual increase was observed after the 10ns simulation time. However, this deviation continues along MD simulation time for complex PPRAס- 
lauric acid, and PPRAס-bis(2-ethylhexyl) phthalate, but in the case of PPRAס-oleanolic acid, dramatically increase throughout MD simulation time as shown in Fig 6. The sudden change in deviation might indicate the high potency of these compounds in complex with PPRAठ protein.

To understand the effect of individual amino acids for all the complexes, including the PPRA bound and unbound ligand complex, we analyzed the root mean square fluctuations (RMSf). Consequently, from the RMS $f$ graph, as shown in Fig 6, it was observed that the RMS $f$ of most residues in all the four systems showed similar fluctuations, but dramatic swing occurs only in the ligand-binding site of the protein, where different compound adopts various favorable interactions with active site residues.
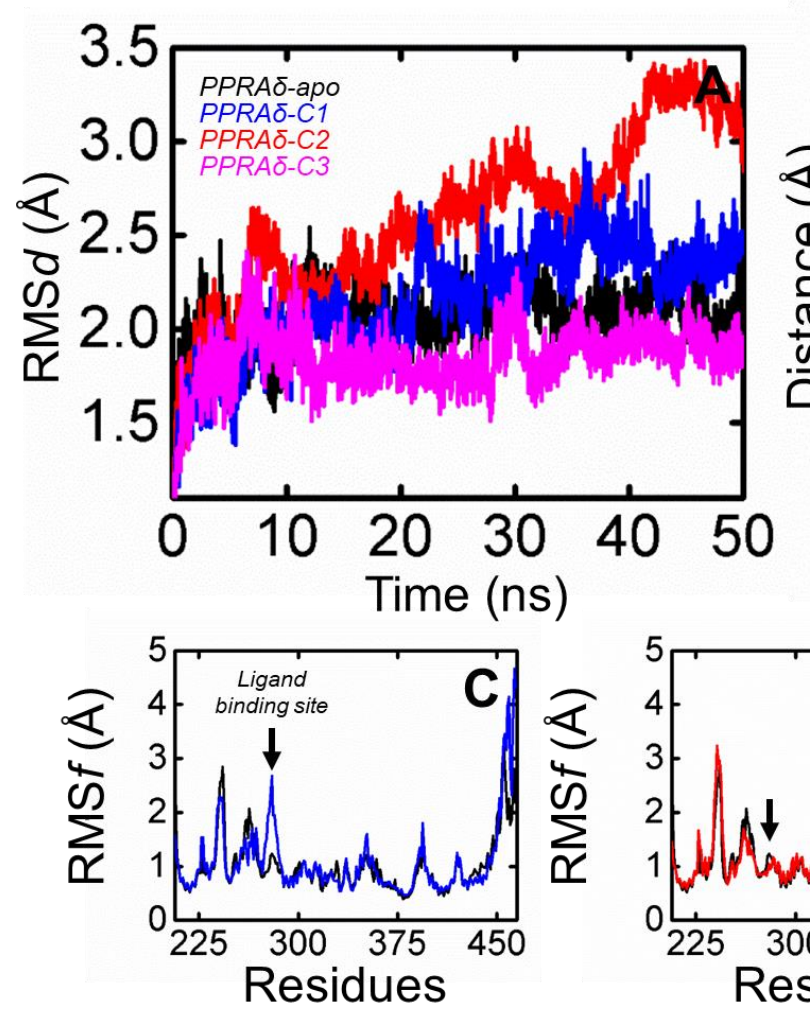

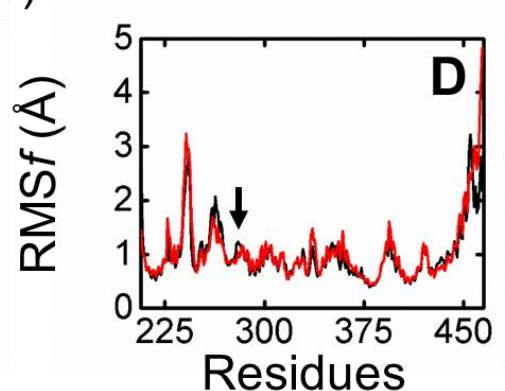

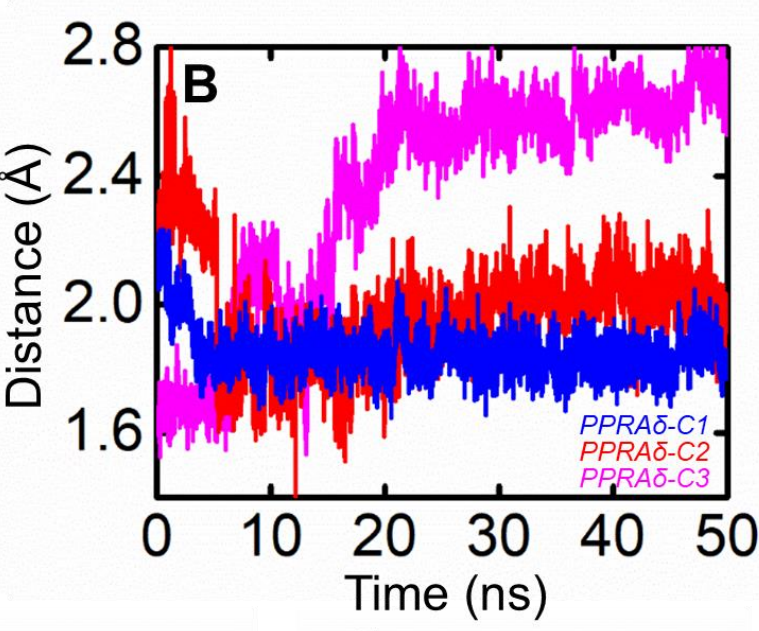

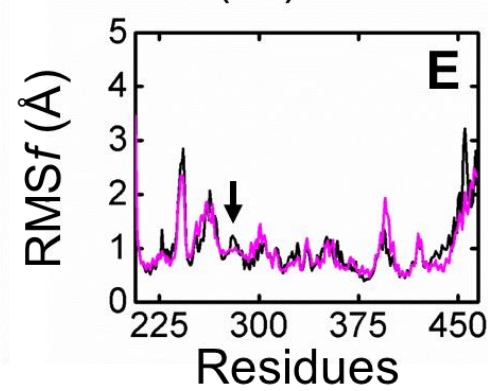

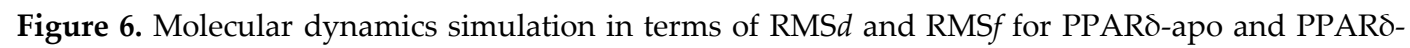
ligand complexes. The distance among the bound ligand and PPRA $\delta$ protein to explore the behavior of the compound in the active pocket concerning MD simulation time. (A) Superposed RMSd graph. (B) Superposed carbon alpha distance graph for APO and bound ligand complex with PPRAঠ protein.

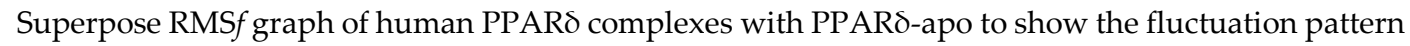
throughout 50ns molecular dynamics simulation time (C) for PPRA $\delta$-apo and C1(Lauric acid), (D) for

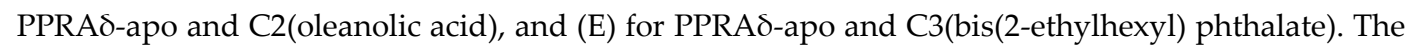
residues are numbering set according to the crystallographic structure.

Generally, from this data, we could assume that PPRAס- Oleanolic acid attained high fluctuation not only in the binding site but additionally in nearby residues, which further supports the high potency of this compounds and clearly illustrate the high profile of ligand-protein interaction profile. While in the case of the other two complexes, in the case of PPRAס-Lauric acid, a slight fluctuation observed but comparatively less than the fluctuation pattern for PPRAס-oleanolic acid. In the case of PPRAठ-bis(2-ethylhexyl) phthalate, slightly weak change found, which further indicates the inhibition pattern of this compound, no doubt this compound attained inhibitory activity again the corresponding protein. More ever, carbon alpha distance analysis was conducted to explore the binding pattern concerning MD simulation time. Subsequently, it was observed that, in the case of

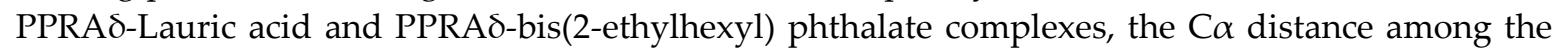
protein and ligand showed comparatively different behaviors like it indicate that concerning MD 
simulation time, the compounds attained various behavior. While in the case of PPRA acid, a consistent behavior among the protein and oleanolic acid was observed, which further suggests that this compound sustains the favorable interactions with the active site residues throughout the MD simulation time.

Additionally, the distance count profile was plotted to show the stability behavior of all the ligand-bound complexes. This graph supports our distance analysis among the protein and ligand, that the PPRAS-oleanolic acid complex attained stable behaviors but other two compounds concerning time attained various behaviors which further indicates the less potency, that regarding MD simulation time these two-compound attained various pattern of interaction, in-out behavior which is inappropriate for the high potency of the corresponding compounds [34-38]. The protein-

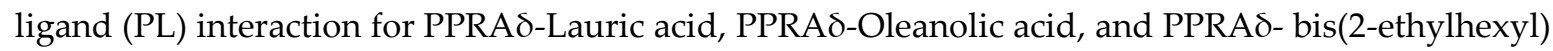
phthalate profiles were depicted in Fig 7.
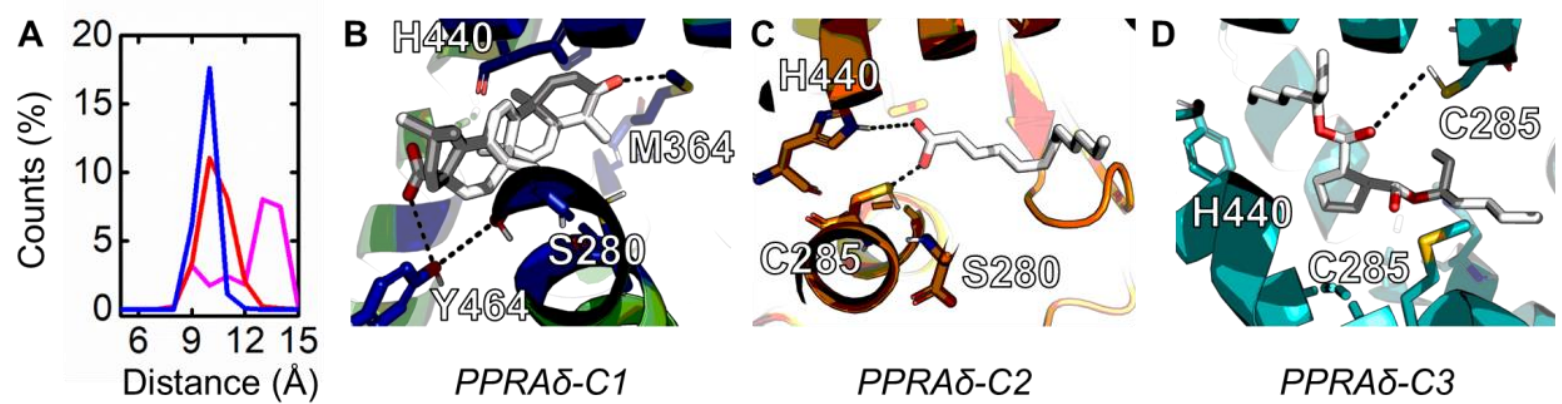

Figure 7. Post-MD simulation extracted structure, protein-ligand interaction, and the carbon alpha distance counts. (a) The carbon alpha distance counts demonstrated that the PPRAS-C2 was more stable regarding distance among the protein and C2(oleanolic acid); it means this C2 showed smoothness in their behavior. In contrast, another C1(Lauric acid) and C3(bis(2-ethylhexyl) phthalate) were highly unstable, change their conformation gradually. The protein-ligand interaction profile for

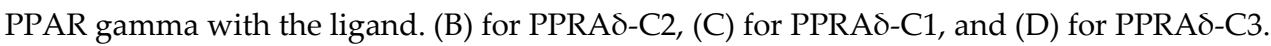

\section{Conclusion}

Hepatocellular carcinoma has the second highest mortality rate among all cancers, thereby obliging the scientific community to start investigating new therapeutic. Natural products are safe and contribute $50 \%$ of the total available therapeutics. The present study revealed that the natural compounds Lauric acid, oleanolic acid, and bis(2-ethylhexyl) phthalate have the anti-proliferative effect, induced apoptosis, and cell death through extrinsic and intrinsic pathways in hepatocellular carcinoma cells. Furthermore, molecular docking and dynamic simulation showed a significant binding affinity of these compounds with the target protein. Hence, owing to further experimental validation, our results may aid in the active treatment of HCC in the future. Moreover, the strategy described herein is of general nature and can also be employed against other carcinomas.

Conflicts of Interest: The authors declare that there are no conflicts of interest.

Author Contributions: SH: BA, and AUR conceived the original idea, $\mathrm{SH}$ performed the experiments, $\mathrm{SH}$ wrote the manuscript, SBJ, BA, MWK, DK, AA, and SU review and approved the manuscript. All authors discussed the results and contributed to the final manuscript.

Acknowledgments: The authors are very thankful to the Higher Education Commission (H.E.C), Islamabad, Pakistan, for the financial support under the National Research Program for Universities (NRPU), Project No. 3334.

\section{References}

1. Amador, M.L., et al., Progress in the development and acquisition of anticancer agents from marine sources. Annals of Oncology, 2003. 14(11): p. 1607-1615.

2. Duke, J.A., et al., Natural products from plants. 2016: CRC press. 
3. Gonelimali, F.D., et al., Antimicrobial properties and mechanism of action of some plant extracts against food pathogens and spoilage microorganisms. Frontiers in microbiology, 2018. 9.

4. $\quad$ Eisenberg, D.M., et al., Unconventional medicine in the United States--prevalence, costs, and patterns of use. New England Journal of Medicine, 1993. 328(4): p. 246-252.

5. Islam, M.S., et al., Antitumor and phytotoxic activities of leaf methanol extract of Oldenlandia diffusa (willd.) roxb. Global J Pharmacol, 2009. 3(2): p. 99-106.

6. Amara, A.A., M. El-Masry, and H. Bogdady, Plant crude extracts could be the solution: extracts showing in vivo antitumorigenic activity. Pakistan journal of pharmaceutical sciences, 2008. 21(2).

7. Abu-Dahab, R. and F. Afifi, Antiproliferative activity of selected medicinal plants of Jordan against a breast adenocarcinoma cell line (MCF7). Scientia Pharmaceutica, 2007. 75(3): p. 121-146.

8. Fatima, N., et al., Biological activities of Rumex dentatus L: Evaluation of methanol and hexane extracts. African Journal of Biotechnology, 2009. 8(24).

9. Mutha, R.E., R.D. Shimpi, and R.B. Jadhav, Study of preliminary anticancer potential of some hemiparasite plants. International Journal of Pharma Research and Development, 2010.

10. Choo, S.P., et al., Comparison of hepatocellular carcinoma in E astern versus W estern populations. Cancer, 2016. 122(22): p. 3430-3446.

11. Golabi, P., et al., Mortality assessment of patients with hepatocellular carcinoma according to underlying disease and treatment modalities. Medicine, 2017. 96(9).

12. Omata, M., et al., Asia-Pacific clinical practice guidelines on the management of hepatocellular carcinoma: a 2017 update. Hepatology international, 2017. 11(4): p. 317-370.

13. Siegel, R.L., K.D. Miller, and A. Jemal, Cancer statistics, 2017. CA: a cancer journal for clinicians, 2017. 67(1): p. 7-30.

14. Hassan, S., et al., In vivo pharmacological investigation of Monotheca buxifolia and Bosea amherstiana using animal models. Saudi Journal of Biological Sciences, 2018.

15. Nasir, E., S. Ali, and R.R. Stewart, Flora of west Pakistan. 1972.

16. Gayathri, L., et al., Hepatotoxic effect of ochratoxin A and citrinin, alone and in combination, and protective effect of vitamin E: In vitro study in HepG2 cell. Food and Chemical Toxicology, 2015. 83: p. 151-163.

17. Farshori, N.N., et al., Cytotoxicity Assessments of Portulaca oleraceaand Petroselinum sativum Seed Extracts on Human Hepatocellular Carcinoma Cells (HepG2). Asian pacific journal of cancer prevention, 2014. 15(16): p. 6633-6638.

18. Cheng, Y., et al., Cisplatin and curcumin co-loaded nano-liposomes for the treatment of hepatocellular carcinoma. International journal of pharmaceutics, 2018. 545(1-2): p. 261-273.

19. Wu, H., et al., Preparation and antitumor evaluation of self-assembling oleanolic acid-loaded Pluronic P105/D$\alpha$-tocopheryl polyethylene glycol succinate mixed micelles for non-small-cell lung cancer treatment. International journal of nanomedicine, 2016. 11: p. 6337.

20. Inc., C.C.G., Molecular operating environment (MOE). 2016, Chemical Computing Group Inc 1010 Sherbooke St. West, Suite\# 910, Montreal ....

21. Cronet, P., et al., Structure of the PPAR $\alpha$ and $\gamma$ ligand binding domain in complex with AZ 242; ligand selectivity and agonist activation in the PPAR family. Structure, 2001. 9(8): p. 699-706.

22. Darden, T., D. York, and L. Pedersen, Particle mesh Ewald: An N. $\log (N)$ method for Ewald sums in large systems. The Journal of chemical physics, 1993. 98(12): p. 10089-10092.

23. Aytenfisu, A.H., et al., Revised RNA dihedral parameters for the Amber force field improve RNA molecular dynamics. Journal of chemical theory and computation, 2017. 13(2): p. 900-915.

24. Berendsen, H.J., D. van der Spoel, and R. van Drunen, GROMACS: a message-passing parallel molecular dynamics implementation. Computer physics communications, 1995. 91(1-3): p. 43-56.

25. Al-Lihaibi, S.S., et al., Three new cembranoid-type diterpenes from Red Sea soft coral Sarcophyton glaucum: Isolation and antiproliferative activity against HepG2 cells. European journal of medicinal chemistry, 2014. 81: p. 314-322.

26. Elshafie, H.S., et al., Cytotoxic activity of Origanum vulgare L. on hepatocellular carcinoma cell line HepG2 and evaluation of its biological activity. Molecules, 2017. 22(9): p. 1435.

27. Zhivotosky, B. and S. Orrenius, Assessment of apoptosis and necrosis by DNA fragmentation and morphological criteria. Current Protocols in Cell Biology, 2001. 12(1): p. 18.3. 1-18.3. 23.

28. Matassov, D., et al., Measurement of apoptosis by DNA fragmentation, in Apoptosis Methods and Protocols. 2004, Springer. p. 1-17.

29. Errami, Y., et al., Apoptotic DNA fragmentation may be a cooperative activity between caspase-activated deoxyribonuclease and the poly (ADP-ribose) polymerase-regulated DNAS1L3, an endoplasmic reticulumlocalized endonuclease that translocates to the nucleus during apoptosis. Journal of Biological Chemistry, 2013. 288(5): p. 3460-3468. 
30. Zhang, M., et al., BAPTA blocks DNA fragmentation and chromatin condensation downstream of caspase-3 and DFF activation in HT-induced apoptosis in HL-60 cells. Apoptosis, 2001. 6(4): p. 291-297.

31. Liu, J., et al., ERK inhibition sensitizes cancer cells to oleanolic acid-induced apoptosis through ERK/Nrf2/ROS pathway. Tumor Biology, 2016. 37(6): p. 8181-8187.

32. Akl, M.R., et al., 3-O-[N-(p-fluorobenzenesulfonyl)-carbamoyl]-oleanolic acid, a semisynthetic analog of oleanolic acid, induces apoptosis in breast cancer cells. Eur J Pharmacol, 2014. 740: p. 209-17.

33. Liu, J., et al., p38 MAPK signaling mediates mitochondrial apoptosis in cancer cells induced by oleanolic acid. Asian Pacific journal of cancer prevention: APJCP, 2014. 15(11): p. 4519-4525.

34. Khan, M.T., et al., Insight into novel clinical mutants of RpsA-S324F, E325K, and G341R of Mycobacterium tuberculosis associated with pyrazinamide resistance. Computational and Structural Biotechnology Journal, 2018. 16: p. 379-387.

35. Qin, F., et al., Induced fit or conformational selection for RNA/U1A folding. Rna, 2010. 16(5): p. 1053-1061.

36. Miller III, B.R., et al., MMPBSA. py: an efficient program for end-state free energy calculations. Journal of chemical theory and computation, 2012. 8(9): p. 3314-3321.

37. Chen, H.-F., Mechanism of coupled folding and binding in the siRNA-PAZ complex. Journal of chemical theory and computation, 2008. 4(8): p. 1360-1368.

38. Wang, J., et al., Reducing grid dependence in finite-difference Poisson-Boltzmann calculations. Journal of chemical theory and computation, 2012. 8(8): p. 2741-2751.

(C) 2020 by the authors. Submitted for possible open access publication under the terms and conditions of the Creative Commons Attribution (CC BY) license (http://creativecommons.org/licenses/by/4.0/). 